\title{
Finding the water refractive index in a swimming pool using a ruler
}

\author{
Jair Lúcio Prados Ribeiro*10 \\ ${ }^{1}$ Instituto Federal de Brasília, Campus Samambaia, Samambaia Sul, Brasília, DF, Brasil
}

Received on March 3, 2020. Accepted on April 6, 2020.

\begin{abstract}
When a swimming pool is filled with water, the tiles covering its walls appear to have smaller vertical dimensions than the real ones, due to the refraction of light from the water into the air. This condition was used to find the refractive index of water, using a trigonometric equation developed from a geometrical model. The necessary measurements were made with a common ruler and the average value obtained for the refractive index was very close to the real one, favoring the didactic potential of this experimental activity.

Keywords: refractive index, refraction, experimental activity.
\end{abstract}

\section{Introduction}

During our last vacation, we were relaxing in a small swimming pool at the hotel where we were staying when we noticed that the tiles covering the opposite wall seemed to have different sizes (Figure 1), although their actual heights were approximately the same. Such an illusion occurs, of course, because of the refraction of light at the surface between water and air. We then wondered whether we could further analyze this everyday situation in order to determine the refractive index of water.

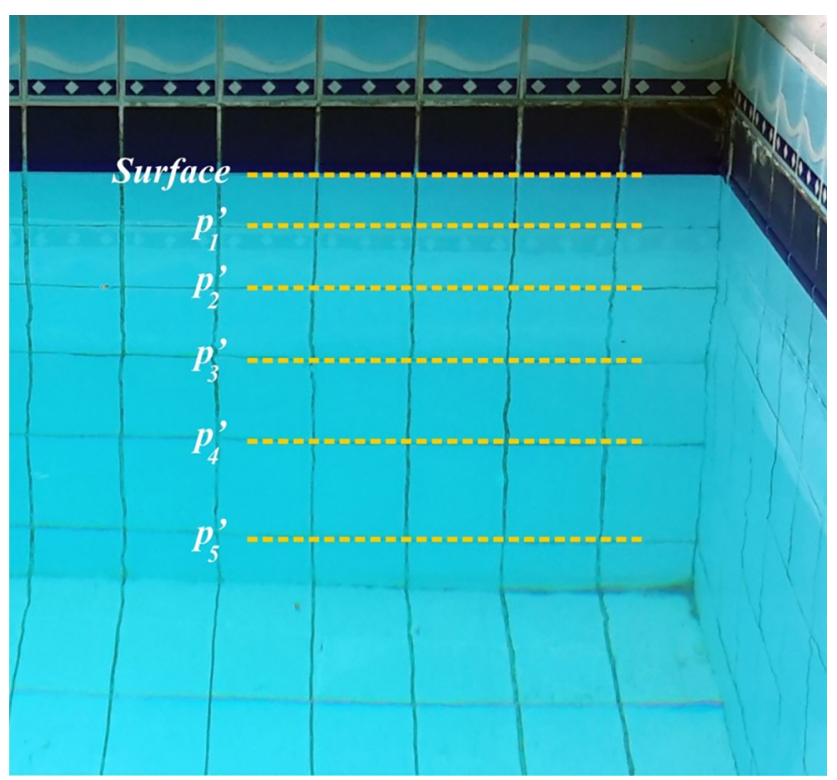

Figure 1: Due to refraction, the pool tiles look smaller to an observer out of the water.

${ }^{*}$ Correspondence email address: jairlucio@gmail.com
Articles published earlier in the literature posit some of the various ways in which water's refractive index can be measured. For example, it is possible to determine this magnitude from the separation of reflected and refracted rays on its surface [1] or using the small-angle approximation to analyze the path of a laser through the liquid [2]. It is also possible to analyze the total internal reflection on a glass "sandwich" immersed in water [3] or to work with the circular diffraction pattern formed when a laser passes through fine particles dissolved in the liquid [4]. A simple refractometer can yet be developed by using a laser pointer and a flat mirror [5] or by an updated version of Pfund's method [6]. A comprehensive review of different methods used for the measurement of refractive indices for various kinds of materials can also be found in Singh [7].

We did not find in these previous articles, however, any mention to the daily situation that we perceived during our relaxing time, which is why we decided to compose this article. Even Kruglak [8], when mentioning an optics experiment developed also in a swimming pool in which water's refractive index was measured, did not cite the occurrence we describe here.

\section{Geometrical and Trigonometrical Description}

This condition occurs due to different refractive indices $(n)$ for water $(n>1)$ and air $(n=1.0)$. Thus, the light rays coming from one of the lines between two tiles (at a depth $p$ ) move away from the normal line when transitioning into the air, causing an observer to perceive a virtual image closer to the surface at an apparent depth $p^{\prime}$, as Figure 2 shows. 


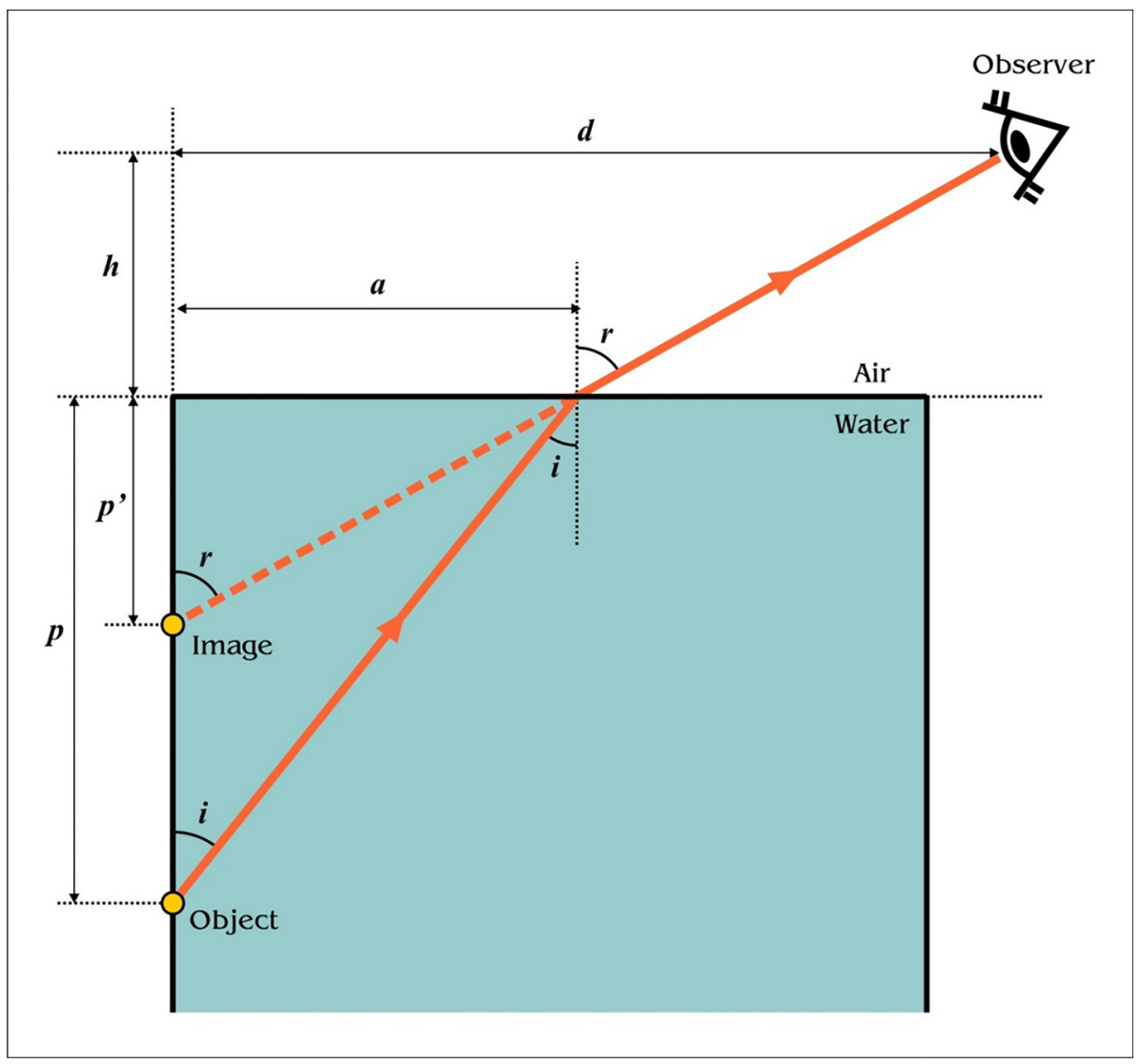

Figure 2: Geometrical diagram displaying the condition and the relevant angles and lengths.

The observer (or a camera, as in the case of the photograph reproduced in Figure 1) is located at the horizontal distance $d$ from the wall and at the height $h$ from the surface of the water. Using Snell's Law:

$$
n \sin i=1 \sin r \rightarrow \frac{1}{n}=\frac{\sin i}{\sin r}
$$

Since both triangles represented in Figure 2 are right triangles, we can write:

$$
\begin{aligned}
& \tan \left(\frac{\pi}{2}-r\right)=\frac{p^{\prime}}{a} \rightarrow a=p^{\prime} \tan r \\
& \tan \left(\frac{\pi}{2}-i\right)=\frac{p}{a} \rightarrow a=p \tan i
\end{aligned}
$$

Dividing equation [2] by equation [3], it comes:

$$
\frac{p^{\prime}}{p}=\frac{\tan i}{\tan r}=\frac{\sin i}{\sin r} \cdot \frac{\cos r}{\cos i}
$$

Using the Pythagorean trigonometric identity:

$$
\frac{\cos r}{\cos i}=\frac{\sqrt{1-\sin ^{2} r}}{\sqrt{1-\sin ^{2} i}}=\frac{\sqrt{1-\sin ^{2} r}}{\sqrt{1-\left(\frac{\sin r}{n}\right)^{2}}}
$$

By replacing the results [1] and [5] in equation [4], we obtain:

$$
\frac{p^{\prime}}{p}=\frac{1}{n} \frac{\sqrt{1-\sin ^{2} r}}{\sqrt{1-\left(\frac{\sin r}{n}\right)^{2}}}
$$

Upon squaring both sides of equation [6], it comes:

$$
\left(\frac{p^{\prime}}{p}\right)^{2}=\frac{1-\sin ^{2} r}{n^{2}-\sin ^{2} r}
$$

Then:

$$
n=\sqrt{\left(\frac{p}{p^{\prime}}\right)^{2} \cdot\left(1-\sin ^{2} r\right)+\sin ^{2} r}
$$

But the sine of the angle of refraction can be calculated by:

$$
\sin r=\frac{d}{\sqrt{d^{2}+\left(h+p^{\prime}\right)^{2}}}
$$

Therefore, replacing the result [9] in equation [8], it follows: 


$$
n=\sqrt{\left(\frac{p}{p^{\prime}}\right)^{2} \cdot\left(1-\frac{d^{2}}{d^{2}+\left(h+p^{\prime}\right)^{2}}\right)+\frac{d^{2}}{d^{2}+\left(h+p^{\prime}\right)^{2}}}
$$

\section{Measurements and Results}

Since we were on vacation, the only measuring instrument we found was a simple ruler borrowed from the hotel receptionist. We then made some measurements of the size of the submerged tiles and found out that each one of them was about a $10 \times 20 \mathrm{~cm}$ rectangle. It is possible to notice in Table 1 that $p$ values are not integer multiples of $20 \mathrm{~cm}$, due to the presence of the separation joints between the tiles. The deeper tiles, which we disregarded in our measurements, were an exception: they had different sizes among them because the depth of the pool near the edge was greater. We also determined with the same ruler the location of the camera in relation to the wall and found $d=1.40 \mathrm{~m}$ and $h=0.50$ $\mathrm{m}$.

The measurements of apparent depth $\left(p^{\prime}\right)$ were made from the picture of the situation itself, as shown in Figure 1 (we kindly asked the receptionist to print the picture 1 for us). Such measures were established by a simple proportion rule: the height of the blue tile that appears just above the surface of the water (see Figure 1) was $10 \mathrm{~cm}$, but in our printed photo this length was $1.8 \mathrm{~cm}$. Using this scale, it was thus possible to determine the five $p^{\prime}$ lengths from the printed figure. The found values were rounded to the second decimal place.

We then placed our experimental values in equation 10 in order to construct table 1 , from which we found $n=1.35$ for the water refractive index.

\section{Final Comments}

We admit that the measurements we performed can display a considerable margin of error due to the improvised conditions in which they were done. Nevertheless, we still ponder that the results are very satisfactory, since 1.35 , our rounded average value for the water refractive index, is very close to the actual refractive index, which is $n=1.333$ [9].

Table 1: Actual depth $(p)$, apparent depth $\left(p^{\prime}\right)$ and refractive index $(n)$.

\begin{tabular}{lll}
\hline $\boldsymbol{p}$ & $\boldsymbol{p}$ & $\boldsymbol{n}$ \\
\hline 0.20 & 0.08 & 1.33 \\
0.41 & 0.17 & 1.37 \\
0.60 & 0.29 & 1.33 \\
0.82 & 0.41 & 1.37 \\
1.01 & 0.56 & 1.34 \\
\hline
\end{tabular}

\footnotetext{
${ }^{1}$ It was necessary to make some little adjustments to Figure 1 before printing in order to measuring the apparent depths $\left(p^{\prime}\right)$ correctly. After all, one can easily perceive that, even in the absence of water, the apparent heights of the tiles would not be constant. This happens because the tiles next to the bottom of the pool would be further from the eye (or camera) then the tiles next to the top, making those next to the bottom look smaller, producing a perspective effect. In order to correct this issue, we used Snapseed, an Android app which has an inbuilt perspective correction tool.
}

However, this great accuracy $(1.2 \%)$ of the value we found in our experimental activity with the actual one can be illusory. A more rigorous look at the measurements indicate that it is not possible to determine the index of refraction to two decimal places from this experimental setup, forcing a new rounding. In this case, the value we obtained is 1.4, differing by more than $5 \%$ from the standard value. Ironically, this result seems more appropriate to us, given the crude measurement procedures we used.

The results, of course, may show lower error margins if more accurate measuring devices are used or if an experimental model can be built in the laboratory (using, for example, an aquarium to simulate the pool and smaller tile coverings to emulate its walls). Our plans include building such an experimental model for educational applications in the classroom, as swimming pools are not part of Brazilian public schools status quo.

In our view, the analysis of everyday situations, such as the one discussed in this article, facilitates students' understanding of physics concepts. We also see this activity with an intrinsic didactic potential for teaching Physics in secondary school, as the necessary measures can be carried out with a very simple instrument (a common ruler). We recognize, however, that the algebraic analysis and trigonometry identities that we present can pose some difficulties, particularly among high school students. Nonetheless, the mathematics is what makes this procedure work and is key to the paper, so we believe it is important that students be guided through the math, since it is worth the effort. Of course the phenomenon should be initially studied from geometrical explanatory diagrams (like Figure 2), but the diagram alone is not sufficient. After all, the whole point of the article (finding water refractive index $n$ ) means expressing it in terms of lengths we can measure with a simple ruler.

\section{References}

[1] J. Kettler, Physics Teacher 32, 190 (1994).

[2] R. Newburgh, W. Rueckner, J. Peidle and D. Goodale, Physics Teacher 38, 478 (2000).

[3] T. Greenslade Jr., Physics Teacher 45, 420 (2007).

[4] S. George and R. Doebler, Physics Teacher 29, 462 (1991).

[5] A. Leung, S. George and R. Doebler, Physics Teacher 29, 226 (1991).

[6] T. Greenslade Jr., Physics Teacher 17, 394 (1979).

[7] S. Singh, Physics Scripta 65, 167 (2002).

[8] H. Kruglak, Physics Teacher 25, 440 (1987).

[9] E. Hetch, Optics (Pearson Education, Londres, 2011), $4^{\text {th }}$ ed., p. 95 . 